INPLASY

PROTOCOL

To cite: Mo et al. Efficacy of combination of Shegan Mahuang Decoction and Montelukast Sodium in the Treatment of Cough Variant Asthma: A Systematic Review and Meta-Analysis. Inplasy protocol 2021120107. doi: 10.37766/inplasy2021.12.0107

Received: 23 December 2021

Published: 23 December 2021

Corresponding author: Yi zhou

zhoumen1123456@163.com

Author Affiliation:

College of Basic

Medicine,Chengdu University of Traditional Chinese

Medicine.

Support: None.

Review Stage at time of this submission: Preliminary searches.

Conflicts of interest: None declared.

\section{Efficacy of combination of Shegan Mahuang Decoction and Montelukast Sodium in the Treatment of Cough Variant Asthma: A Systematic Review and Meta-Analysis}

\author{
Mo, J1; Zhang, Y2; Han, L3; Tang, $\mathrm{H}^{4}$.
}

Review question / Objective: Is combination of shegan mahuang decoction and montelukast sodium effective for cough variant asthma?Can combination of shegan mahuang decoction and montelukast sodium be a complementary and alternative therapy for cough variant asthma?P: Participant: Patients diagnosed with cough variant asthma; I: Intervention: combination of shegan mahuang decoction and montelukast sodiu; O:Outcome: effective rate; S: Study design: RCTs.

Condition being studied: Cough variant asthma (CVA) is special type of asthma, cough is the only or mainly clinical manifestation. There are no obvious symptoms or signs such as wheezing and shortness of breath, but the airway is highly responsive. At present ,the treatment of CVA is mainly included inhale glucocorticoid and bronchodilator with oral corticosteroid and leukotriene regulator.

INPLASY registration number: This protocol was registered with the International Platform of Registered Systematic Review and Meta-Analysis Protocols (INPLASY) on 23 December 2021 and was last updated on 23 December 2021 (registration number INPLASY2021120107).

\section{INTRODUCTION}

Review question / Objective: Is combination of shegan mahuang decoction and montelukast sodium effective for cough variant asthma?Can combination of shegan mahuang decoction and montelukast sodium be a complementary and alternative therapy for cough variant asthma?P: Participant: Patients diagnosed with cough variant asthma; I: Intervention: combination of shegan mahuang decoction 
and montelukast sodiu; 0:Outcome: effective rate; S: Study design: $\mathrm{RCTs.}$

Condition being studied: Cough variant asthma (CVA) is special type of asthma, cough is the only or mainly clinical manifestation. There are no obvious symptoms or signs such as wheezing and shortness of breath, but the airway is highly responsive. At present, the treatment of CVA is mainly included inhale glucocorticoid and bronchodilator with oral corticosteroid and leukotriene regulator.

\section{METHODS}

Participant or population: Patients who were diagnosed with cough variant asthma (as diagnosed by a clinician, or using any recognized diagnostic criteria). In the same study, patients in different intervention groups had no significant imbalance in baseline conditions such as age, gender and course of disease, and were comparable.

Intervention: The treatment group received combination of shegan mahuang decoction and montelukast sodium therapies.

\section{Comparator: Montelukast sodium.}

Study designs to be included: Randomized controlled trials (RCTS) of combination of shegan mahuang decoction and montelukast sodium therapies for the treatment of cough variant asthma, regardless of the time of publication or field of study, regardless of whether hidden or blind allocation was involved. Nonrandomized controlled trials, such as animal trials, reviews, conferences and papers, clinical case reports, and personal experience, are excluded.

Eligibility criteria: The inclusion criteria were prespecified as types of participants:patients diagnosed with cough variant asthma using any recognized diagnostic criteria,regardless of age, gender, source of cases,duration of disease, ethnicity, or nationality;(2) types of interventions: combination of shegan mahuang decoction and montelukast sodium clearly stated in the trial group either alone or in combination with other treatments;no restriction was imposed on the administration mode, dosage, and course of treatment; (3) types of comparator(s)/control: patients treated with montelukast sodium; (4) types of outcome measures: The main effective rate, recurrence rate, rate of adverse reaction,FEV1,ect. (5) types of study: RCT. Exclusion criteria included (1) nonRCTs, reviews, animal-based research, conference proceedings, and literature review; (2) unclear diagnostic criteria and outcome measurements; (3) unable to get original data; (4) duplicated publications; and (5) other CM treatments involving acupuncture and massage.

Information sources: The Cochrane Central Register of Controlled Trials (CENTRAL), PubMed, EMBASE, CBM,CNKI,VIP, WanFang will be searched for relevant information, updated to December 2021 without any restriction of language or publication date. To achieve comprehensive information, we will contact with authors, check the trial registers, or search greyliterature.

Main outcome(s): The main effective rate,recurrence rate, ate of adverse reaction,FEV1,ect.The minor outcome is effective rate of main symptoms : 1.IL-4. 2.Cough time. 3.TNF-a.

Quality assessment / Risk of bias analysis: Two researchers independently evaluated the risk of bias in randomized controlled trials in accordance with the Cochrane Handbook of Systematic Reviewers, including the following items: random sequence generation, allocation concealment, blinding of participants and personnel, blinding of outcome assessment, incomplete outcome data, selective reporting, and other bias. The quality of studies was classified as being at of high, unclear, or low risk of bias. After completion, they would recheck. In case of a disagreement, they would discuss. If no agreement could be reached, a decision woud be made in consutation with researchers from the third party. 
Strategy of data synthesis: For each trial,we will present outcome data as point estimates with mean and standard deviation for continuous outcomes and risk ratios (RRs) with corresponding $95 \%$ confidence intervals (Cis for dichotomous outcomes. RevMan will be used for direct meta-analysis. Using the Review Manager Software (Revman 5.4). We used fixed-effect meta-analysis if trails were sufficiently similar with no significant heterogeneity. If we had found heterogeneity, we planned to explore the reason by sensitivity analysis followed by random-effect if required. For dichotomous data, we presented the primary outcome of remission of cough variant asthma using risk ratios (RR) with $95 \%$ confidence intervals (Cl). For continuous data, we would calculate mean difference if the results were measured in the same way between trails. If studies used different methods to assess the same outcome, we would have calculated standardized mean difference to combine trails. As to assessment of reporting biases, we would have generated a funnel plot and visually inspected for asymmetry when the studies are more than ten. Publication bias also was determined using Egger's test to funnel plot asymmetry.

Subgroup analysis: When there is disagreement in the results, a subgroup analysis needs to be carried out for different reasons. Heterogeneity is mainly manifested in many aspects such as race, sex, age, drug formulations, different forms of intervention, treatment time, and drug dosages.

Sensitivity analysis: In order to ensure the Credibility of the research results, we will conduct a sensitivity analysls of the included literature and will eliminate low quality literature.

Country(ies) involved: China.

Keywords: shegan mahuang decoction; montelukast sodium; cough variant asthma; systematic review; meta-analysis.
Contributions of each author:

Author 1 - Jingwen MO.

Author 2 - Yiyi Zhang.

Author 3 - Lingling Han.

Author 4 - Huiling Tang. 